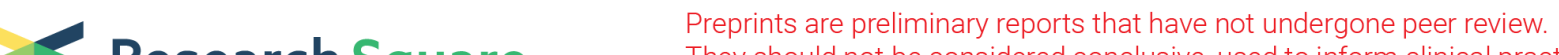 Research Square They should not be considered conclusive, used to inform clinical practice, or referenced by the media as validated information.
}

\section{Systemic Proteomic Analysis Reveals Distinct Exosomal Proteins Profiles in Rheumatoid Arthritis}

\section{Qiu Qin}

Shanghai Pudong New District Zhoupu Hospital

\section{Ronghua SONG}

Shanghai Pudong New District Zhoupu Hospital

\section{Peng DU}

Shanghai Pudong New District Zhoupu Hospital

Chaoqun GAO

Shanghai Pudong New District Zhoupu Hospital

\section{Qiuming YAO}

Shanghai Pudong New District Zhoupu Hospital

Jinan ZHANG ( $\nabla$ zhangjinan@hotmail.com)

Shanghai Pudong New District Zhoupu Hospital

\section{Research article}

Keywords: Rheumatoid arthritis, Exosomes, Differentially expressed proteins, Nanoscale liquid chromatography coupled to tandem mass spectrometry

Posted Date: October 27th, 2020

DOI: https://doi.org/10.21203/rs.3.rs-95581/v1

License: (c) (i) This work is licensed under a Creative Commons Attribution 4.0 International License.

Read Full License 


\section{Abstract}

\section{Background}

Rheumatoid arthritis (RA) is a heterogeneous and complex disease characterized by autoantibodies production and inflammation of the synovium. Its underlying mechanisms remain elusive. In recent years, exosomes have emerged as non-invasive biomarkers in diverse diseases. Due to its various inflammatory and immunological functions, some studies highlighted abnormalities of exosomes in RA, but fewer have paid attention to the broad spectrum of potential systemic markers of RA. The aim of this study was to identify exosomal candidate proteins in the pathogenesis of RA using systemic proteomic method.

Methods

Totally 12 specimens of plasma from 6 RA patients and 6 age-and gender-matched controls from the Chinese population were obtained for nanoscale liquid chromatography coupled to tandem mass spectrometry (nano-LC-MS/MS) analysis to identify differentially expressed proteins (DEPs) in exosomes. Gene ontology (GO), protein interaction network (PPI) and KEGG pathway analyses were used in subcellular localization analysis.

Results

A total of 278 exosomal proteins were detected. Among them, 32 proteins were significantly upregulated ( $F C \geq 2.0$ and $P<0.05$ ) and 5 proteins were downregulated ( $F C \leq 0.5$ and $P<0.05$ ). Most of these DEPs were immunoglobullins (Igs). Bioinformatics analysis indicated that these Igs are involved in inflammatory response of RA through diverse immune regulatory pathways, such as NF-kappa B signaling pathway. PPI analysis revealed that transthyretin (TTR, P02766), angiotensinogen (AGT, P01019), lipopolysaccharide-binding protein (LBP, P18428), monocyte differentiation antigen CD14 (CD14, P08571), cartilage oligomeric matrix protein (COMP, P49747), serum amyloid P (SAP/APCS, P02743) and tenascin (TNC, P24821) can interact with each other or may form a regulatory networks. Subsequently, these cross-linked proteins may mainly involve in the NF-kappa B signaling pathway or inflammatoryrelated pathways to mediate the onset of RA. Noteworthy, among these exosomal DEPs, the complex composed of LBP and CD14 is involved in NF-kappa B signaling pathway, resulting in promoted expression of IL-8, TNF-a, and eventually leading to the development of RA.

\section{Conclusions}

Our findings suggest distinct plasmic exosomal protein profiles in RA patients. These proteins not only take important parts in the vicious circle in the pathogenic process of RA, but also serve as novel biomarkers in RA diagnosis and prognosis.

\section{Background}


Rheumatoid arthritis (RA) is the most common systemic autoimmune disorder affecting approximately $0.5-1 \%$ of the adult population worldwide. The disease is characterized by inflammation and proliferation of synovial tissue, destruction of cartilage and bone, and production of various autoantibodies ${ }^{[1]}$ and often presents systemic manifestations including eye, skin, pulmonary, peripheral neuropathy and cardiovascular events during progression ${ }^{[2]}$. With its prevalence constantly rising, it has become one of the main causes of disability in human and requires enormous public health resources for treatment, prevention and sequelae amelioration.

The pathogenesis of RA involves communication between synovia cells and infiltrated immune cells such as T cell, B cell and synovial fibroblast. The clinical picture of RA is the result of a close interaction between cells, soluble mediators, autoantibodies and signal transduction pathways of the innate and adaptive immune system. Various studies have been conducted to find different biomarkers in disease development. Since the first description of rheumatoid factor (RF) as one of the diagnostic criteria in 1940, anti-cyclic citrullinated peptide antibody (ACPA) has been discovered as an accurate diagnostic and prognostic index for RA with a higher specificity but lower sensitivity. Currently, the diagnosis of RA is mainly based on the clinical symptoms, imaging results and some traditional laboratory tests. These methods are often used to diagnose RA during the middle-to-late disease period when treatments are unable to effectively control the disease progression ${ }^{[3]}$. Furthermore, as a complex and heterogeneous disease, about $30-40 \%$ of RA patients do not have a good response to an optimal dosing regimen of drugs including methotrexate (MTX). With the advent of biologic therapies, more prognostic markers have been used to help stratify patients for suitable treatments. In general, more efficient biomarkers need to be explored for early diagnosis and treatment of RA.

Exosomes are stable nanometer-sized $(50-100 \mathrm{~nm})$ membranous extracellular vesicles secreted from different type of cells to a variety of biological fluids, including blood, urine and synovial fluids. Exosomes can deliver varieties of proteins, lipids, DNA and RNA both locally and distantly and interact with cells to participate intercellular communication ${ }^{[4]}$. Because of their small size, stability, biologically active content and specific targeting, exosomes, as a natural delivery system, may become a promising therapeutic modality. In addition, their carry-on contents not only reflect the physiological or pathological conditions of parental cells, but also can mediate intercellular communication and functionally alter their recipient cells ${ }^{[5]}$. Unlike other conventional delivery systems, exosomes can potentially avoid degradation, go through barriers and deliver cargo directly into the cytoplasm ${ }^{[6]}$. Moreover, exosomes secreted by brain tumor cells carry disease-specific, immunosuppressive and carcinogenic factors ${ }^{[7]}$. Recent studies have shown that exosomes are involved in inflammatory processes of cancer, inflammatory bowel diseases, type 2 diabetes mellitus, obesity and neurodegenerative diseases. Although few studies have revealed the abnormal expression of exosomes in RA patients, research on their function in RA still remains in its infancy. Hence, in this study, we implemented a case-control study to explore the proteomic profiling of plasma-derived exosomes in RA patients.

\section{Materials And Methods}




\section{Sample collection}

Blood samples were obtained from 6 RA patients and 6 gender- and age-matched healthy controls from the Outpatient Clinic of the Endocrinology Department of Zhoupu Hospital (Shanghai, China). All participants were Chinese in origin. Clinical and demographic data are shown in Table 1. RA patients were diagnosed according to the criteria by 2010 ACR/EULAR. None of the patients had received therapy. The controls showed negative RF, ACAP, inflammatory indexes (such as ESR, CRP, SAA) and had no concomitant with other inflammatory and autoimmune diseases. Whole blood samples were centrifuged at $4{ }^{\circ} \mathrm{C}$ at $1500 \mathrm{~g}$ for $10 \mathrm{~min}$ and at $2000 \mathrm{~g}$ for $20 \mathrm{~min}$ to separate out plasma. All plasma samples were stored at $-80^{\circ} \mathrm{C}$ until use.

All subjects received written informed consent for participation. The study was approved by the Ethics Committee of Zhoupu Hospital.

\section{Exosomal proteins separation and quality control}

Plasma exosomes were extracted using the magnetic beads sorting method. Briefly, $1 \mathrm{ml}$ plasma was mixed with $5 \mathrm{ml}$ magnetic bead. After washed for three times, exosomal proteins were lysed using Lysis Buffer 3 containing $1 \mathrm{mM}$ phenylmethylsulfonyl fluorid (PMSF) and $2 \mathrm{mM}$ ethylene diamine tetraacetic acid (EDTA). After votexing (vortex-genie 2, Shanghai, China) and standing for $5 \mathrm{~min}$, dithiothreitol (DTT) was added into the mixture to final concentration of $10 \mathrm{mM}$. The mixture was then centrifuged at 25,000 $\mathrm{g}$ for $20 \mathrm{~min}$ at $4^{\circ} \mathrm{C}$ and the supernatant was collected. After incubated at $56^{\circ} \mathrm{C}$ for $1 \mathrm{~h}$, the proteins were precipitated by adding four times volume of cold acetone and incubating at room temperature for $45 \mathrm{~min}$ in the dark. After centrifugation at $25,000 \mathrm{~g}$ for $20 \mathrm{~min}$, the pellets were collected and resuspended in $\mathrm{X}$ buffer. The concentration of plasma exosomal proteins were measured using the Bradford assay (BioRad, Hercules, CA). The integrity quality control of the collected proteins was verified by protein electrophoresis on $12 \%$ uniform SDS-polyacrylamide gels using the Ettan DALT II system (Amersham Bioscience, Uppsala, Sweden) at $120 \mathrm{~V}$ for $120 \mathrm{~min}$. The separated proteins were visualized by Coomassie blue staining.

\section{Exosomal proteins enzymolysis}

Approximately $100 \mu \mathrm{g}$ of the extracted exosomal proteins were digested using trypsin (1:40 w/w, Promega, Madison, USA) at $37^{\circ} \mathrm{C}$ for $12 \mathrm{~h}$. The peptides were further purified using Strata-X-C solid phase extraction system (Phenomenex, Torrance, USA), lyophilized by speed-vacuum to remove the reaction solvents, and dissolved in $20 \mathrm{mM} \mathrm{NH}_{4} \mathrm{FA}, \mathrm{pH} 10$ for the following experiments.

4. High pH reversed-phase high performance liquid chromatography (Hi-pH RP HPLC)

A total of $200 \mu \mathrm{g}$ mixed exosomal proteins were separated by high-pH RP HPLC. Basically, peptides diluted in solution $\mathrm{A}(5 \% \mathrm{ACN}, \mathrm{pH}$ 9.8) were injected onto a high-pH RP column (Luna C18 column with inner diameter of $4.6 \mathrm{~mm}$ and length of $250 \mathrm{~mm}$, Phenomenex, CA, USA), and eluted by step linear elution 
program, that is 0-10 min fast elution from $5 \%$ of solution $\mathrm{B}(95 \% \mathrm{CAN}, \mathrm{pH} 9.8), 10-40$ min linear elution from $5 \%-35 \%$ of solution $B$, and $40-41$ min washing elution from $35 \%-95 \%$ of solution $B$. The separation was performed in a Prominence HPLC system (Shimadzu, Nakagyo-ku, Kyoto, Japan) at the flow rate of $1.0 \mathrm{~mL} / \mathrm{min}$ with monitoring wavelength of $214 \mathrm{~nm}$ and collection of one component per minute. At last, 10 fractions of peptides isolated by RP method were lyophilized.

The labeled peptides were dissolved in $0.1 \%$ formic acid (FA) solution. The supernatants were collected after centrifugation at 20,000 $\mathrm{g}$ for $10 \mathrm{~min}$. The samples were injected onto an UltiMate 3000 UHPLC (Thermo Fisher Scientific, Waltham, MA, USA) trap column (150 $\mu \mathrm{m}$ i.d., C18 $1.8 \mu \mathrm{m} \times 25 \mathrm{~cm}$ ) and eluted at a flow rate of $500 \mathrm{ml} / \mathrm{min}$ using the following gradient method: 0-5 min with mobile phase of $5 \%$ solution $B(98 \% A C N, 0.1 \% F A), 5-160$ min with $5 \%-35 \%$ solution $B, 160-170$ min with $35 \%-80 \%$ solution $B$, 170-175 min with $80 \%$ solution $B$, and $176-180$ min with $5 \%$ solution $B$.

5. Nanoscale liquid chromatography coupled to tandem mass spectrometry (nano-LC-MS/MS) analysis

\subsection{Data-dependent acquisition (DDA) MS analysis}

The peptides separated by liquid phase were delivered onto a nanoESI system setting in the positive ion mode. Then the elutes were directly entered Q-Exactive HF (Thermo Fisher Scientific, San Jose, CA) to conduct DDA with primary MS scan from 350-1500 m/z at resolution of 60,000, and MS/MS scan at 100 $\mathrm{m} / \mathrm{z}$ with resolution of 15,000 . The screening conditions for the second-stage fragmented precursor were set as ions peak intensity exceeding 10,000 and top 20 precursor ions. The raw MS/MS data were converted into MGF format by Proteome Discoverer 1.2 (Thermo Fisher Scientific, Waltham, MA, USA), and searched by Mascot 2.3.02 (Matrix Science, Boston, MA, USA) against the UniprotKB/Swiss-Prot human protein database (http://www.uniprot.org).

\subsection{Data-independent acquisition (DIA) MS analysis}

The peptides separated by liquid phase were delivered onto a nanoESI system setting in the positive ion mode. Then the elutes were directly entered Q-Exactive HF (Thermo Fisher Scientific, San Jose, CA) to conduct DIA with primary MS scan from 350-1500 m/z at resolution of 120,000 and fragments with 350$1500 \mathrm{Da}$ were divided into 40 windows for fragmentation and signal acquisition. The DIA data were analyzed using Spectronaut software and the bioinformatics analysis was simultaneously performed in this process.

After data processing for relative quantification of exosomal proteins, proteins with fold change $(\mathrm{FC})>2.0$ and $P$ value $<0.05$ were considered as significant differentially expressed proteins (DEPs).

\section{Statistical analysis}

The statistical significance of differences in parameters of clinical features between the RA and controls was calculated by Student's t-test. The statistical significance of differences in exosomal proteins 
intensity between the 2 groups was calculated by a one-way analysis of variance. A $p$ value $<0.05$ was considered statistically significant.

\section{Results}

\section{Demographic characteristics and clinical features}

The clinical and serological features of the 6 RA patients ( 2 males and 4 females) are shown in Table 1. Their mean age was $54 \pm 9.1$ years, ranging from 43 to 66 years. The mean age of the 6 age- and gendermatched healthy controls ( 2 males and 4 females) was $53.5 \pm 7.48$ years, ranging from 40 to 60 years. All RA patients had elevated RF and ACPA levels and no one has received any medication.

\section{The unique proteins profiles of plasma-derived exosomes}

A total of 278 exosomal proteins were detected in all samples with high confidence. Among them, 37 were differentially expressed. In detail, 32 were upregulated and 5 were downregulated in RA patients $(p<0.05)$. Although most upregulated proteins were related to immunoglobulins (Igs), some were closely related to inflammatory or autoimmune diseases, including monocyte differentiation antigen CD14 (CD14), lipopolysaccharide-binding protein (LBP), serum amyloid P-component (SAP/APCS), tenascin (TNC) and cartilage oligomeric matrix protein (COMP). Table 2 and Figure 1 show the detailed information on unique proteins profiles in plasma exosomes of RA patients.

The subcellular localization analysis of these 37 DEPs using WoLF PSORT software (https://psort.hgc.jp/) showed that 28 were positioned at extracellular (extr), 3 located in nucleus (nucl), 2 located in mitochondria (mito) and 2 cyto_nucleus (cyto_nucl), and 1 located in plasma membrane (plas) and 1 endoplasmic reticulum (ER) respectively.

\section{Gene Ontology (GO) enrichment of DEPs}

To obtain a global image of the proteomic changes during RA development, the DEPs were annotated with GO terms using the DAVID database (https://david.ncifcrf.gov/), based on the biological processes (BP), molecular functions (MF), and cellular component (CC). For the classification of BP, the top 10 enriched annotation terms were cellular process, biological regulation, regulation of biological process, metabolic process, response to stimulus, cellular component organization or biogenesis, positiveregulation of biological process, multicellular organismal process, negative regulation of biological process, immune system process (Fig. 2A). These proteins have varies functions including binding, catalytic activity, molecular function regulator, molecular transducer activity, structural molecule activity as well as signal transducer activity (Fig.2B) and are cellular components of organelle, extracellular region, cell, cell part, extracellular region part, membrane, organelle part, macromolecular complex, membrane-enclosed lumen, membrane part, cell junction, and supramolecular complex (Fig.2B). Impressively, from a immune-regulated point of view based on BP annotation, the significantly upregulated DEPs are enriched in the lipopolysaccharide-mediated signaling pathway and regulation of toll- 
like receptor signaling pathway, as shown in Fig. 2A1. Besides, other inflammatory pathways that are significantly associated with DEPs, including leukocyte activation and IL-8 production, were also found uncommonly elevated in RA patients (data not shown).

\section{Protein-protein interaction network (PPI)}

Protein-protein associations of these DEPs were identified using the STRING Protein Library (https://string-db.org/) and the resulted network interaction map of the top 100 credible data were drafted and shown in Figure 3. Some unique exosomal proteins were found interacting with each other or forming regulatory networks, including LBP (P18428), CD14 (P08571), COMP (P49747), TNC (P24821), SAP (P02743), transthyretin (TTR, P02766) and angiotensinogen (AGT, P01019). These results are compatible with $\mathrm{GO}$ analysis, indicated that these exosomal proteins may be essential for the development of RA or have multiplicative effects on the onset of RA.

\section{Pathway analysis}

Pathway analysis was conducted based on the latest KEGG (Kyoto Encyclopedia of Genes and Genomes, http://www.genome.jp/kegg/) database. Almost all the DEPs up-regulated in RA patients are involved in staphylococcus aureus infection, NF-kappa B signaling pathway, phagosome, PI3K/Akt signaling pathway, tuberculosis, natural killer cell mediated cytotoxicity, B cell receptor signaling pathway, systemic lupus erythematosus (SLE), autoimmune thyroid disease (AITD), RA, and other inflammation and immune-related pathways. The five DEPs downregulated in RA proteins are involved in amoebiasis and SLE (Fig. 4).

Integrated metabolic pathways analysis and pathways network relationship prompted that the topranking pathway with DEPs between RA patients and healthy controls was NF-kappa B signaling pathway. Additionally, in this study, both increased LBP (P18428) and CD14 (P08571) were involved in this pathway (ko04064, details are shown in Figs. 5 and 6).

\section{Discussion}

RA is a chronic disease with unknown causes. The exact entity of RA is still too complicated to be fully understood. Recently, exosomes have been attracting tremendous attention due to its powerful capability to deliver signals both intercellularly and intracellularly. As all we know that a single diagnostic biomarker is not sufficient to reflect altered body environment, especially for a heterogeneous and complex diseases such as RA. Multi-biomarkers are important for the diagnosis and monitoring of these diseases ${ }^{[8]}$. Proteomics tools allow identifying global proteins and comparing protein expression patterns. In addition, proteomic analysis of protein-protein interactions and networks facilitates the identification of new proteins correlating with known proteins. In order to provide in-depth insights into the system-level molecular mechanisms of circulating exosomes in RA, the nano-LC-MS/MS method, a more sensitive proteomic technique, was used to detect plasma exosomal proteins differentially expressed between RA patients and normal controls. 
We exhibited a specific circulating exsomal-protein profile with proteins enriched in inflammatory and immunoregulatory pathways. Among the total 278 exosomal proteins detected, 32 proteins were significantly upregulated and 5 were downregulated. Most of these DEPs were Igs. Further bioinformatics analysis combining $\mathrm{GO}$ and pathway analysis indicated that these lgs are involved in inflammatory responses of RA etiology through diverse immune regulatory pathways, such as NF-kappa B signaling pathway, Fc epsilon RI signaling pathway, primary immunodeficiency or B cell receptor signaling pathway. Exosomes from this study as well as others showed some common signatures, such as COMP, but not exactly matched. Protein-protein interaction network analysis found some unique proteins profiles in RA patients. These proteins include TTR, AGT, LBP, CD14, COMP, SAP and TNC and can interact with each other or combine to form complexes.

Among these DEPs, COMP was the most upregulated one with already affirmed importance role in the pathogenesis of RA. COMP is primarily expressed in cartilage and numerous other tissues/cells, including synovium, ligaments, fibroblasts, and vascular smooth muscle cells ${ }^{[9]}$ and known to involve in collagen secretion and fibrillogenesis and interact with various other components of the extracellular matrix (ECM) [10]. The pathophysiological process in RA progression involves the digestion and dissolution of the intercellular components of the connective tissue by protease-derived hydrolysis. As a cartilage-derived marker of cartilage breakdown, COMP is undoubtedly considered as a prognostic factor in RA, especially at early stage. For instance, serum COMP level is significantly higher in patients with RA compared with control subjects, and its predictive accuracy is much higher than elevated ACPA level as a biomarker ${ }^{[11]}$. Moreover, both serum and synovial COMP levels are significantly correlated with the early-stage RA, especially the synovial COMP level, which increases more than twofold compared to the serum level [12]. Globally, most studies have shown that serum COMP level is higher in RA patients ${ }^{[13,14]}$. Consistent with these studies, our data also showed elevated COMP protein level in RA patients compared to normal controls. The pathway analysis found that elevated COMP might participate in the pathogenesis of RA. Our data highlight the promising potential role of COMP as a prognostic biomarker in RA. Noteworthy is that COMP is released due to catabolic reaction and high turnover of cartilage matrix in RA. Therefore, it can be not only used for RA diagnosis, but also for monitoring disease progression in patients with more advanced joint cartilage damage.

We also found up-regulated levels of LBP-CD14 complex in RA patients. Previous studies have reported crosstalk between LBP and CD14. LBP extracts LPS from bacterial membranes, binds and transfers LPS to CD14, and delivers it to the Toll-like receptor 4-myeloid differentiation 2 (TLR4-MD2) complex, which finally leads to activation of multiple signaling components and subsequent production of proinflammatory cytokines of immune cells ${ }^{[15]}$. Emerging evidences have confirmed the aforementioned proinflammatory and immune effects of LBP. For example, circulating LBP concentration is significantly higher in patients with juvenile idiopathic arthritis (JIA) than controls ${ }^{[16]}$. Plasmatic LBP and sTLR4 are associated with knee osteoarthritis progression ${ }^{[17]}$. In addition, LBP has been suggested as a new marker of synovial inflammation, and its level is significantly higher in RA patients ${ }^{[18,19]}$. Moreover, LBP has also been proposed as a sensitive serum biomarker to evaluate RA disease activity ${ }^{[20]}$. CD14 has both 
conventional proinflammatory and unconventional anti-inflammatory effects in various diseases. For example, CD14 exerts significant proinflammatory impacts in the pathogenesis of metabolic diseases, such as obesity and diabetes mellitus ${ }^{[21]}$. Conversely, CD14 reduces the severity of intestinal lesions and ulcerations ${ }^{[22]}$, demonstrating its protective role in autoimmune diseases. The terminal effects of CD14 in inflammation depend on many factors, such as nature of the disease, CD14 expression level, inflammation site, and competition among different CD14-dependent pathways. In line with most previous studies, we found overexpression of LBP-CD14 complex in RA patients. It is not surprising to hypothesize that increased LBP and CD14 catalyze LPS transfer to the TLR/MD2 (ko04620) complex, which can greatly activates NF-kappa B (ko04064) signaling pathway, promoting the expression of IL-8, TNF- $a$ and finally inducing the occurrence of RA.

In addition to COMP, TNC is another exosomal-protein closely related to the pathogenesis of RA. Numerous studies on arthritic animal models and RA patients have confirmed higher TNC density in RA. In murine arthritic models, TNC is arthritogenic following its intra-articular infection and its expression is required to maintain chronic joint inflammation ${ }^{[23]}$. In patients with RA, TNC level is elevated in both RA cartilage and synovium and soluble TNC form is detectable in synovial fluids. Additionally, high TNC is associated with more erosive joint disease and predicts poor response to biological treatment in RA patients ${ }^{[24]}$. Noteworthy, a citrullinated peptide from the fibrinogen-like globe domain of TNC is detected in RA synovial fluids, and antibodies to cyclic peptides containing citrullinated sites are found in both preRA and RA sera, suggesting the important role of TNC in both RA diagnosis and prediction ${ }^{[25]}$. Our data revealed TNC expression is elevated in RA patients possibly via ECM-receptor interaction pathway (ko04512) and TNC may regulate expression of miRNAs (k005206) involved in the pathogenesis of RA. Blocking proinflammatory signals from the matrix to change the synovial microenvironment, such as TNC antagonist, may ameliorate the progression of RA without engendering global immune suppression.

SAP is another exosomal protein with multiple characteristics. SAP can bind to various molecules, then engulfed by phagocytic cells. SAP expression tends to be lowered in diverse diseases including pulmonary fibrosis, myelofibrosis, RA, and mixed connective tissue diseases, indicating that SAP deficiency might be involved in part of fibrosis ${ }^{[26,27]}$. Few researches indicated that SAP is elevated in morbidly obese children ${ }^{[28]}$, suggesting that SAP also has a proinflammatory effect. In our research, plasma SAP is enhanced in RA patients. Considering the results of other published studies, we speculate that exposure of RA-related autoantigens to immune system could lead to release chromatin, which then binds to SAP, being isolated from the antigens and subsequently driving immune responses.

Our PPI analysis also indicated that TTR and AGT participate in the protein-protein interaction network in RA patients, which is barely reported previously. TTR is increased in RA, OA and JIA ${ }^{[29]}$. Although the mechanism underlying the enhanced TTR is not well understood, evidence has shown that TTR is one of the targets attacked by autoantibodies. AGT is reported to regulate the expression of proinflammatory transcription factor NF-kappa B ${ }^{[30]}$. 
Overall, we globally mapped a distinct exosomal protein profile in RA patients and indicated that these exosomes exert an immunoregulated action to trigger the development of RA. Therefore, they might be useful biomarkers complementary to RF and ACPA to, at least, partially reflect the existence and development of RA and used for RA diagnose by easily and simultaneously detecting them in a single sample with high sensitivity and specificity.

Although our sample size is sufficient to show significant differences among the clinical groups, it should be admitted that the potential application of exosomal protein profile identified in this study need to be verified in other studies with large sample size using other molecular methods.

\section{Conclusion}

In summary, our data once again demonstrated the important role of inflammatory processes in the pathogenesis of RA. Deep bioinformatics data analysis for the first time revealed the interactions among LBP, CD14, TNC, COMP, SAP, TTR and AGT and suggested that the interaction among exosomes may play key roles in maintaining the homeostasis of the immune system-synovium axis. The complex composed with LBP and CD14 may participate NF-kappa B signaling pathway, promoting the expression of IL-8 and TNF- $a$ and ultimately leading to the pathogenesis of RA. Even though further studies are needed to elucidate these points, we expect that multi-exosomes founded in our study have potential application in RA diagnosis and can serve as non-invasive biomarkers.

\section{Abbreviations}


Rheumatoid arthritis

RA

Nanoscale liquid chromatography coupled to tandem mass spectrometry nano-LC-MS/MS

Differentially expressed proteins

DEPs

Gene ontology

$\mathrm{GO}$

Protein interaction network

PPI

Transthyretin

TTR

Angiotensinogen

AGT

Lipopolysaccharide-binding protein

LBP

Monocyte differentiation antigen CD14

CD14

Cartilage oligomeric matrix protein

COMP

Serum amyloid $\mathrm{P}$

SAP/APCS

Tenascin

TNC

Anti-cyclic citrullinated peptide antibody

ACPA

Rheumatoid factor

RF

Methotrexate

MTX

Phenylmethylsulfonyl fluorid

PMSF

Ethylene diamine tetraacetic acid

EDTA

Dithiothreitol

DTT

Formic acid

FA

Data-dependent acquisition

DDA

Data-independent acquisition

DIA

Fold change

FC

Extracellular

extr

Nucleus

nucl

Mitochondria

mito

Cyto_nucleus

cyto_nucl

Plasma membrane

plas

Endoplasmic reticulum

ER 


\begin{tabular}{ll} 
Biological processes & BP \\
\hline Molecular functions & MF \\
\hline Cellular component & CC \\
\hline Kyoto Encyclopedia of Genes and Genomes & KEGG \\
\hline Systemic lupus erythematosus & SLE \\
\hline Autoimmune thyroid disease & AITD \\
\hline Extracellular matrix & ECM \\
\hline Toll-like receptor 4-myeloid differentiation 2 & TLR4-MD2 \\
\hline Juvenile idiopathic arthritis & JIA
\end{tabular}

\section{Declarations}

1. Ethics approval and consent to participate

All subjects received written informed consent for participation. The study was approved by the Ethics Committee of Zhoupu Hospital.

2. Consent for publication

Not applicable.

\section{Availability of data and materials}

The datasets used and/or analysed during the current study are available from the corresponding author on reasonable request.

\section{Competing interests}

The authors declare that they have no competing interests.

\section{Funding}

This study was supported by the National Natural Science Foundation of China (No. 81900710).

\section{Authors' contributions}

QQ performed the proteomics examination in RA patients and normal controls, and was a major contributor in writing the manuscript. SRH, DP, GCQ and YQM were involved in the cellection of specimens in this study. ZJA is the corresponding author of this article. All authors read and approved the final manuscript. 
7. Acknowledgements

Not applicable.

\section{References}

[1] McInnes IB, Schett G. The pathogenesis of rheumatoid arthritis. N Engl J Med. 2011; 365(23):220519.

[2] Khanna R, Dlouhy BJ, Smith ZA, Lam SK, Koski TR, Dahdaleh NS. The impact of steroids, methotrexate, and biologics on clinical and radiographic outcomes in patients with rheumatoid arthritis undergoing fusions at the craniovertebral junction. J Craniovertebr Junction Spine. 2015; 6(2):60-4.

[3] Mølbaek K, Hørslev-Petersen K and Primdahl J. Diagnostic delay in rheumatoid arthritis: a qualitative study of symptom interpretation before the first visit to the doctor. Musculoskeletal Care. 2016; 14(1): 26-36.

[4] Record M, Subra C, Silvente-Poirot S, Poirot M. Exosomes as intercellular signalosomes and pharmacological effectors. Biochem Pharmacol. 2011; 81(10):1171-82.

[5] Barile L, Vassalli G. Exosomes: Therapy delivery tools and biomarkers of diseases. Pharmacol Ther. 2017; 174:63-78.

[6] Jiang L, Vader P, Schiffelers RM. Extracellular vesicles for nucleic acid delivery: progress and prospects for safe RNA-based gene therapy. Gene Ther. 2017; 24(3):157-166.

[7] Vos KEVD, Balaj L, Skog J, Breakefield XO. Brain tumor microvesicles: insights into intercellular communication in the nervous system. Cell Mol Neurobiol. 2011;31(6):949-59.

[8] Lee J, Joo EJ, Lim HJ, Park JM, Lee KY, Park A, Seok A, Lee H, Kang HG. Proteomic analysis of serum from patients with major depressive disorder to compare their depressive and remission statuses.

Psychiatry Investig. 2015; 12(2): 249-59.

[9] Posey KL, Coustry F, Hecht JT. Cartilage oligomeric matrix protein: COMPopathies and beyond. Matrix Biol. 2018; 71-72:161-173.

[10] Saberi Hosnijeh F, Siebuhr AS, Uitterlinden AG, Oei EH, Hofman A, Karsdal MA, Bierma-Zeinstra SM, Bay-Jensen AC, van Meurs JB. Association between biomarkers of tissue inflammation and progression of osteoarthritis: Evidence from the Rotterdam study cohort. Arthritis Res Ther. 2016; 18:81.

[11] Liu F, Wang X, Zhang X, Ren C, Xin J. Role of serum cartilage oligomeric matrix protein (COMP) in the diagnosis of rheumatoid arthritis (RA): a case-control study. J Int Med Res. 2016; 44(4):940-9. 
[12] El Defrawy AO, Gheita TA, Raslan HM, El Ansary MM, El Awar AH. Serum and synovial cartilage oligomeric matrix protein levels in early and established rheumatoid arthritis. Z Rheumatol. 2016; 75(9):917-23.

[13] Saghafi M, Khodashahi M, Saadati N, Azarian A, Rezaieyazdi Z, Salehi M, Sahebari M. Relationship between cartilage oligomeric matrix protein (COMP) and rheumatoid arthritis severity. Electron Physician. 2017; 9(12):5940-7.

[14] Sakthiswary R, Rajalingam S, Hussein H, Sridharan R, Asrul AW. Cartilage oligomeric matrix protein (COMP) in rheumatoid arthritis and its correlation with sonographic knee cartilage thickness and disease activity. Clin Rheumatol. 2017; 36(12):2683-8.

[15] Tobias PS, Soldau K, Gegner JA, Mintz D, Ulevitch RJ. Lipopolysaccharide binding protein-mediated complexation of lipopolysaccharide with soluble CD14. J Biol Chem. 1995; 270(18):10482-8.

[16] Fotis L, Shaikh N, Baszis KW, Samson CM, Lev-Tzion R, French AR, Tarr PI. Serologic evidence of gutdriven systemic inflammation in Juvenile idiopathic arthritis. J Rheumatol. 2017; 44(11):1624-31.

[17] Huang ZY, Perry E, Huebner JL, Katz B, Li YJ, Kraus VB. Biomarkers of inflammation-LBP and TLRpredict progression of knee osteoarthritis in the DOXY clinical trial. Osteoarthritis Cartilage. 2018; 26(12):1658-65.

[18] Lee J, Mun S, Kim D, Lee YR, Sheen DH, Ihm C, Lee SH, Kang HG. Proteomic analysis for verification of Rheumatoid Arthritis biomarker candidates using multiple reaction monitoring. Proteomics Clin Appl. 2019; 13(3):e1899911.

[19] Kim D, Mun S, Lee J, Park A, Seok A, Chun YT, Kang HG. Proteomics analysis reveals differential pattern of widespread protein expression and novel role of histidine-rich glycoprotein and lipopolysaccharide-binding protein in rheumatoid arthritis. Int J Biol Macromol. 2018; 109:704-10.

[20] Wen W, Li Y, Cheng Y, He J, Jia R, Li C, Guo J, Sun X, Li Z. Lipopolysaccharide-binding protein is a sensitive disease activity biomarker for rheumatoid arthritis. Clin Exp Rheumatol. 2018; 36(2):233-40.

[21] Fernández-Real JM, Pérez del Pulgar S, Luche E, Moreno-Navarrete JM, Waget A, Serino M, Sorianello E, Sánchez-Pla A, Pontaque FC, Vendrell J, Chacón MR, Ricart W, Burcelin R, Zorzano A. CD14 modulates inflammation-driven insulin resistance. Diabetes. 2011; 60(8): 2179-86.

[22] Buchheister S, Buettner M, Basic M, Noack A, Breves G, Buchen B, Keubler LM, Becker C, Bleich A. CD14 plays a protective role in experimental inflammatory bowel disease by enhancing intestinal barrier function. Am J Pathol. 2017; 187(5): 1106-20.

[23] Midwood KS, Orend G. The role of tenascin-C in tissue injury and tumorigenesis. J Cell Commun Signal 2009; 3(3-4):287-310. 
[24] Page TH, Charles PJ, Piccinini AM, Nicolaidou V, Taylor PC, Midwood KS. Raised circulating tenascin$C$ in rheumatoid arthritis. Arthritis Res Ther. 2012; 14(6): R260.

[25] Schwenzer A, Jiang X, Mikuls TR, Payne JB, Sayles HR, Quirke AM, Kessler BM, Fischer R, Venables PJ, Lundberg K, Midwood KS. Identification of an immunodominant peptide from citrullinated tenascin-C as a major target for autoantibodies in rheumatoid arthritis. Ann Rheum Dis. 2016; 75(10):1876-83.

[26] Murray LA, Chen Q, Kramer MS, Hesson DP, Argentieri RL, Peng X, Gulati M, Homer RJ, Russell T, van Rooijen N, Elias JA, Hogaboam CM, Herzog EL. TGF-beta driven lung fibrosis is macrophage dependent and blocked by serum amyloid P. Int J Biochem Cell Biol. 2011; 43(1):154-62.

[27] Verstovsek S, Manshouri T, Pilling D, Bueso-Ramos CE, Newberry KJ, Prijic S, Knez L, Bozinovic K, Harris DM, Spaeth EL, Post SM, Multani AS, Rampal RK, Ahn J, Levine RL, Creighton CJ, Kantarjian HM, Estrov Z. Role of neoplastic monocyte-derived fibrocytes in primary myelofibrosis. J Exp Med. 2016; 213(9):1723-40.

[28] Anwer M, Lqbal MJ. Serum amyloid P and endocrine markers in a cohort of obese children. Indian J Endocrinol Metab. 2018; 22(5):683-8.

[29] Ni M, Wei W, Feng Q, Sun XG, Wang YC, Gu YJ, Zheng F. Transthyretin as a poential serological marker for the diagnosis of patients with early rheumatoid arthtitis. Clin Exp Rheumatol. 2013; 31(3): 3949.

[30] Okamura A, Rakugi H, Ohishi M, Yanagitani Y, Takiuchi S, Moriguchi K, Fennessy PA, Higaki J, Ogihara T. Upregulation of renin-angiotensin system during differentiation of monocytes to macrophages. J Hypertens. 1999; 17(4): 537-45.

[31] Nahmod KA, Vermeulen ME, Raiden S, Salamone G, Gamberale R, Fernandez-Calotti P, Alvarez A, Nahmod V, Giordano M, Geffner JR. Control of dendritic cell differentiation by angiotensin $\otimes$. FASEB J. 2003; 17(3): 491-3.

\section{Tables}

Table 1 Demographic and clinical data of patients with rheumatoid arthritis and healthy controls. 


\begin{tabular}{|llll|}
\hline Parameter & $\begin{array}{l}\text { RA } \\
\text { patients }(\mathrm{n}=6)\end{array}$ & $\begin{array}{l}\text { Normal } \\
\text { controls }(\mathrm{n}=6)\end{array}$ & Normal range \\
\hline Age(years; $\mathrm{m} \pm \mathrm{s})$ & $54 \pm 9.1$ & $53.5 \pm 7.48$ & \\
\hline Sex(males/females) & $2 / 4$ & $2 / 4$ & \\
\hline Disease duration(months) & $17.83 \pm 19.31$ & $/$ & $0-15.91 \mathrm{U} / \mathrm{ml}$ \\
\hline Rheumatoid factor (RF) & $289.23 \pm 421.36$ & $/$ & $<17 \mathrm{U} / \mathrm{ml}$ \\
\hline $\begin{array}{l}\text { Anti-cyclic citrullinated peptide antibody } \\
\text { ACPA) }\end{array}$ & $398.2 \pm 449.83$ & $/$ & \\
\hline Tender joint count & $4.33 \pm 3.01$ & $/$ & $(3.5-$ \\
\hline Swollen joint count & $2.83 \pm 1.72$ & $/$ & $9.5) \times 10^{9} / \mathrm{L}$ \\
\hline White blood cell (WBC) & $7.15 \pm 2.41$ & $6.9 \pm 0.43$ & $(1.8-6.3)$ \\
\hline Neutrophil (N) & & & $\times 10^{9} / \mathrm{L}$ \\
\hline Erythrocyte sedimentation rate (ESR) & $37.83 \pm 30.09$ & $13 \pm 2.61$ & $0-20 \mathrm{~mm} / \mathrm{h}$ \\
\hline Serum amyloid A (SAA) & $27.62 \pm 29.40$ & $5.88 \pm 2.14$ & $0-10 \mathrm{mg} / \mathrm{L}$ \\
\hline C-reactive protein (CRP) & $19.13 \pm 26.04$ & $5.67 \pm 2.23$ & $0-10 \mathrm{mg} / \mathrm{L}$ \\
\hline Prealbumin (PA) & $234.5 \pm 95.49$ & $308.67 \pm 24.16$ & $220-400 \mathrm{ng} / \mathrm{L}$ \\
\hline
\end{tabular}

Table 2 The significantly differentially expressed exosomal proteins in rheumatoid arthritis. 


\begin{tabular}{|c|c|c|c|}
\hline Protein name & Accession number & $\log 2 \mathrm{FC}^{*}$ & $p$-value \\
\hline \multicolumn{4}{|l|}{ Up-regulated } \\
\hline Immunoglobulin lambda variable 6-57(IGLV6-57) & P01721 & 1.00 & 0.02 \\
\hline Immunoglobulin heavy variable 4-28(IGHV4-28) & A0A0C4DH34 & 1.00 & 0.04 \\
\hline Serum amyloid P-component (SAP/APCS) & P02743 & 1.05 & 0.00 \\
\hline Immunoglobulin lambda-like polypeptide 1(IGLL1) & P15814 & 1.12 & 0.00 \\
\hline Immunoglobulin lambda variable 1-47(IGLV1-47) & P01700 & 1.13 & 0.01 \\
\hline Immunoglobulin heavy constant gamma $2(\mathrm{IGHG} 2)$ & P01859 & 1.15 & 0.01 \\
\hline Complement $\mathrm{C} 1 \mathrm{q}$ subcomponent subunit $\mathrm{A}(\mathrm{C} 1 \mathrm{QA})$ & P02745 & 1.26 & 0.01 \\
\hline Immunoglobulin heavy variable 3-53(IGHV3-53) & P01767 & 1.30 & 0.04 \\
\hline Immunoglobulin heavy variable 3-49(IGHV3-49) & A0A0A0MS15 & 1.36 & 0.01 \\
\hline Midasin OS(MDN1) & Q9NU22 & 1.38 & 0.01 \\
\hline Immunoglobulin heavy variable 1-58(IGHV1-58) & A0A0C4DH39 & 1.44 & 0.05 \\
\hline Coagulation factor $\mathrm{XI}(\mathrm{F} 11)$ & P03951 & 1.51 & 0.01 \\
\hline Immunoglobulin heavy variable 1-8(IGHV1-8) & P0DP01 & 1.63 & 0.03 \\
\hline Inter-alpha-trypsin inhibitor heavy chain $\mathrm{H} 3(\mathrm{ITIH} 3)$ & Q06033 & 1.70 & 0.00 \\
\hline Complement factor I(CFI) & P05156 & 1.70 & 0.00 \\
\hline Immunoglobulin heavy variable 1-18(IGHV1-18) & A0A0C4DH31 & 1.75 & 0.00 \\
\hline Lipopolysaccharide-binding protein(LBP) & P18428 & 1.78 & 0.01 \\
\hline Immunoglobulin lambda variable 1-40(IGLV1-40) & P01703 & 1.82 & 0.00 \\
\hline Immunoglobulin lambda variable 1-36(IGLV1-36) & A0A0B4J1U3 & 1.96 & 0.00 \\
\hline CDKN2A-interacting protein OS(CDKN2AIP) & Q9NXV6 & 1.97 & 0.04 \\
\hline Monocyte differentiation antigen CD14(CD14) & P08571 & 1.97 & 0.01 \\
\hline Immunoglobulin gamma-1 heavy chain (IGY1HC) & P0DOX5 & 2.02 & 0.00 \\
\hline Immunoglobulin heavy constant gamma 3(IGHG3) & P01860 & 2.06 & 0.00 \\
\hline Immunoglobulin heavy variable 4-34(IGHV4-34) & P06331 & 2.09 & 0.00 \\
\hline Immunoglobulin heavy variable 1-69D(IGHV1-69D) & AOA0B4J2HO & 2.20 & 0.01 \\
\hline Immunoglobulin lambda variable 3-1(IGLV3-1) & P01715 & 2.20 & 0.01 \\
\hline Immunoglobulin lambda variable 3-19(IGLV3-19) & P01714 & 2.29 & 0.00 \\
\hline
\end{tabular}




\begin{tabular}{|llll|}
\hline Complement factor H-related protein 5(CFHR5) & Q9BXR6 & 2.37 & 0.03 \\
\hline Prospero homeobox protein 1 (PROX1) & Q92786 & 2.39 & 0.04 \\
\hline Tenmascin(TNC) & A0A0B4J1V2 & 2.42 & 0.03 \\
\hline Cartilage oligomeric matrix protein(COMP) & P24821 & 2.75 & 0.03 \\
\hline Down-regulated & & 2.92 & 0.03 \\
\hline Alpha-actinin-1(ACTN1) & P12814 & -30.34 & 0.00 \\
\hline Transthyretin(TTR) & P02766 & -1.71 & 0.00 \\
\hline Angiotensinogen(AGT) & P01019 & -1.19 & 0.00 \\
\hline Serum paraoxonase/arylesterase 1(PON1) & P27169 & -1.16 & 0.01 \\
\hline Keratin, type I cytoskeletal 9 (KRT9) & P35527 & -1.04 & 0.01 \\
\hline
\end{tabular}

*FC: fold change

\section{Figures}



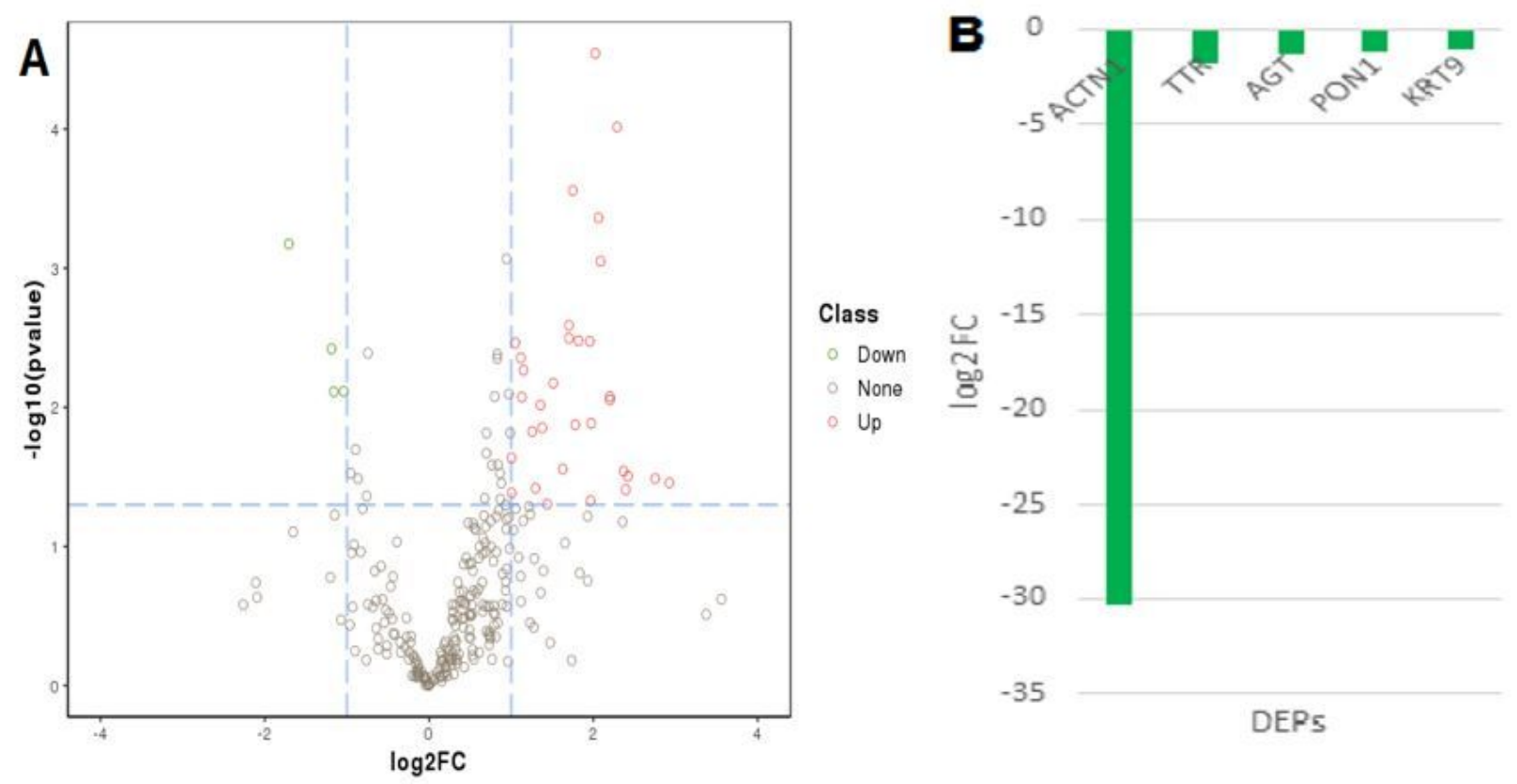

C 3.5

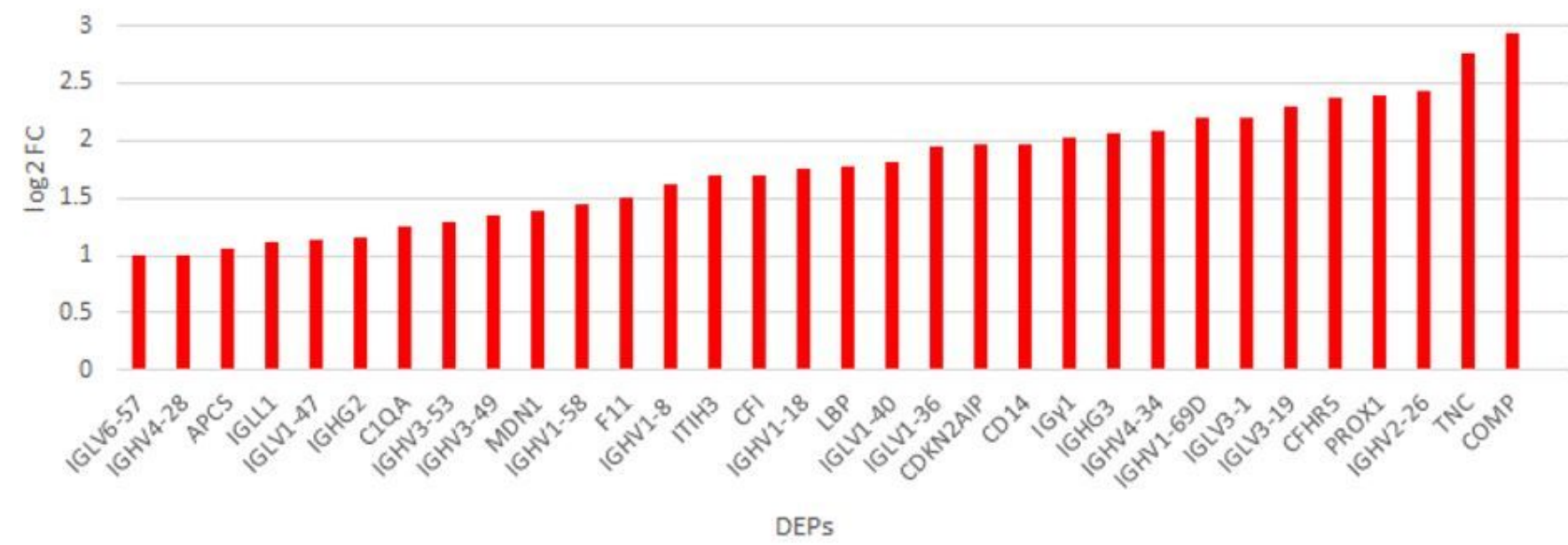

Figure 1

The exosomal proteins profiles in rheumatoid arthritis patients. A) Volcano plots of differentially expressed proteins (DEPS) based on protein abundance changes and t test. (FC: fold change; Red circle: upregulated proteins; Green circle: down-regulated proteins; Grey circle: no difference.) B) The downregulated DEPs. C) The up-regulated DEPs. Details for abbreviated protein names are shown in Table 2. 

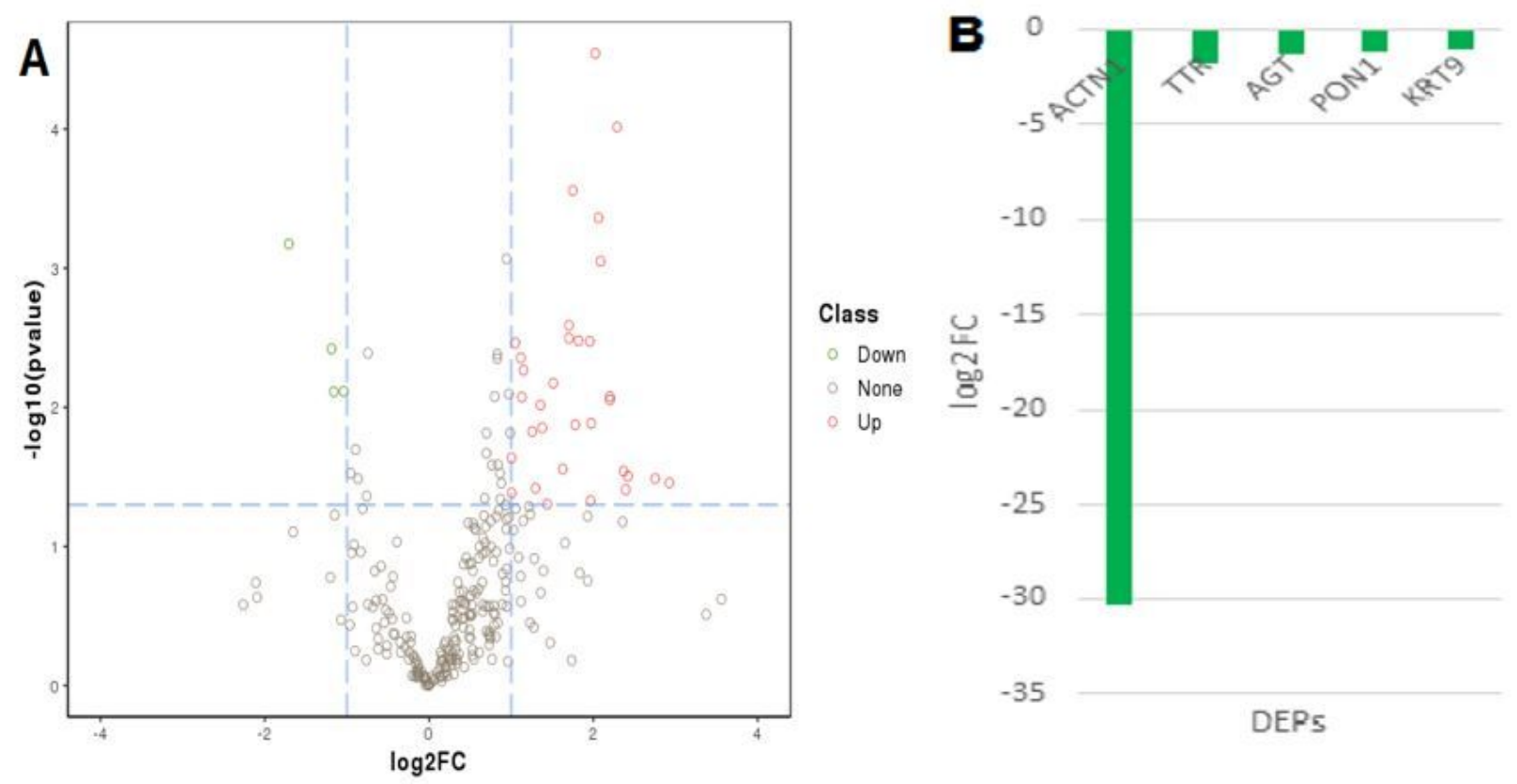

C 3.5

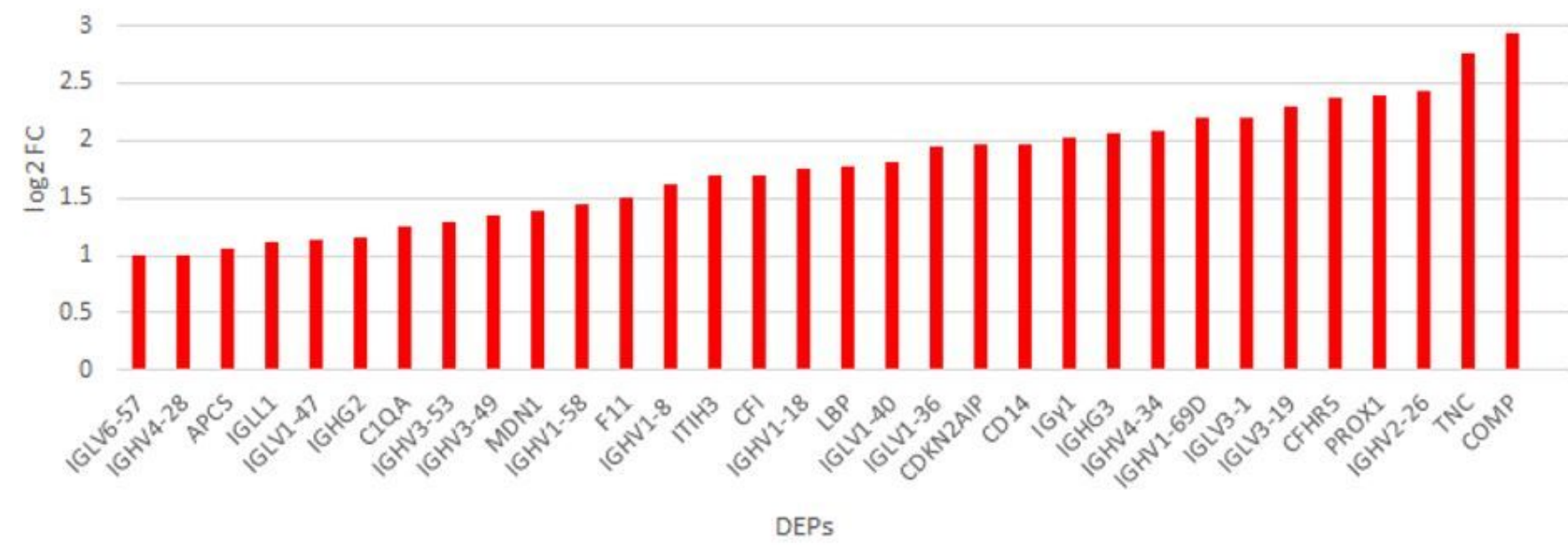

Figure 1

The exosomal proteins profiles in rheumatoid arthritis patients. A) Volcano plots of differentially expressed proteins (DEPS) based on protein abundance changes and t test. (FC: fold change; Red circle: upregulated proteins; Green circle: down-regulated proteins; Grey circle: no difference.) B) The downregulated DEPs. C) The up-regulated DEPs. Details for abbreviated protein names are shown in Table 2. 

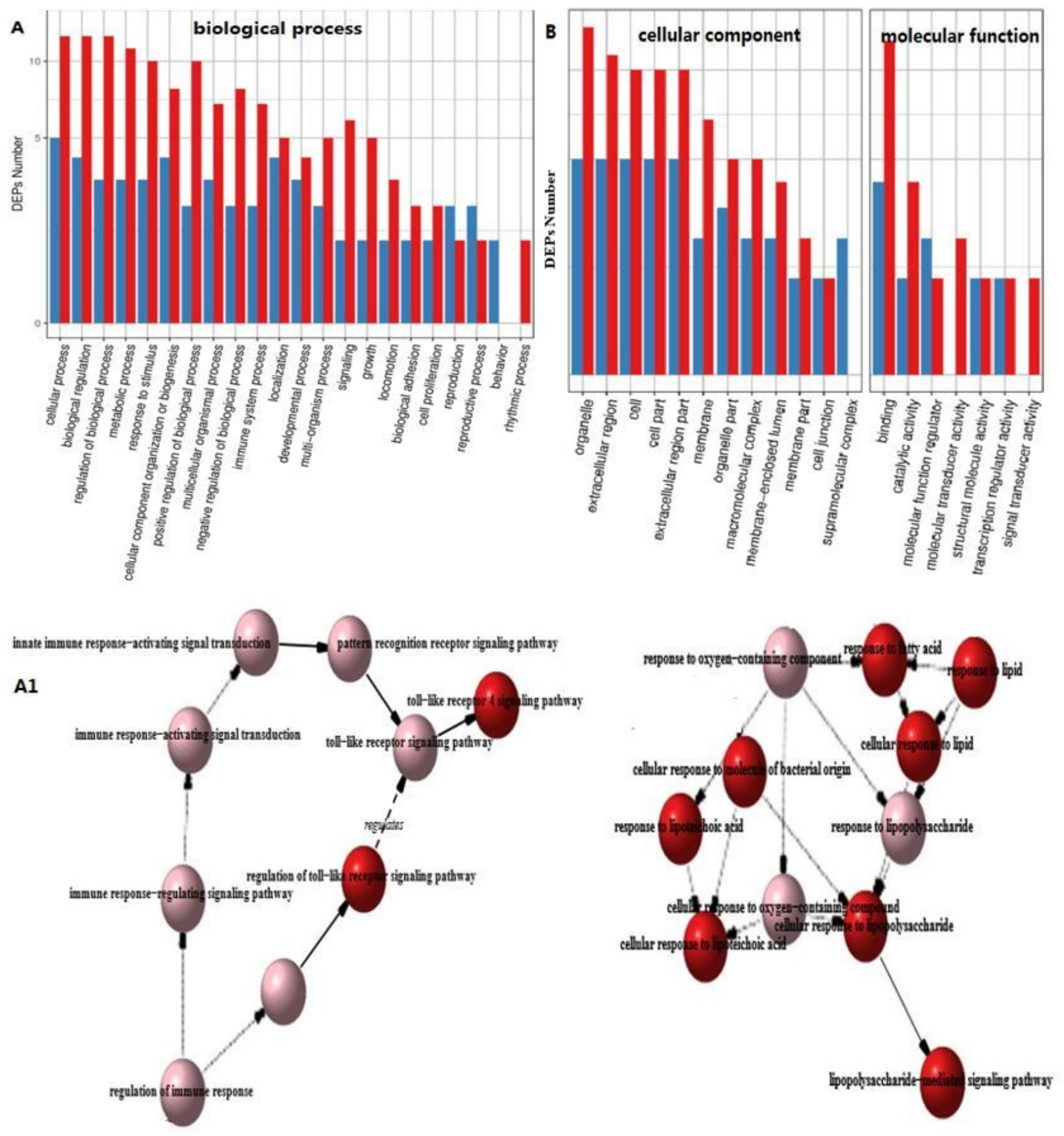

Figure 2

Gene ontology functional classification of plasma-derived exosomes in rheumatoid arthritis. A: Classification of biological process, where red histogram represents upregulated proteins and blue histogram represents downregulated proteins. B: Classification of cellular component and molecular function. A1: The major immune-related networks based on biological process annotation, where red balls represent upregulated pathway. 

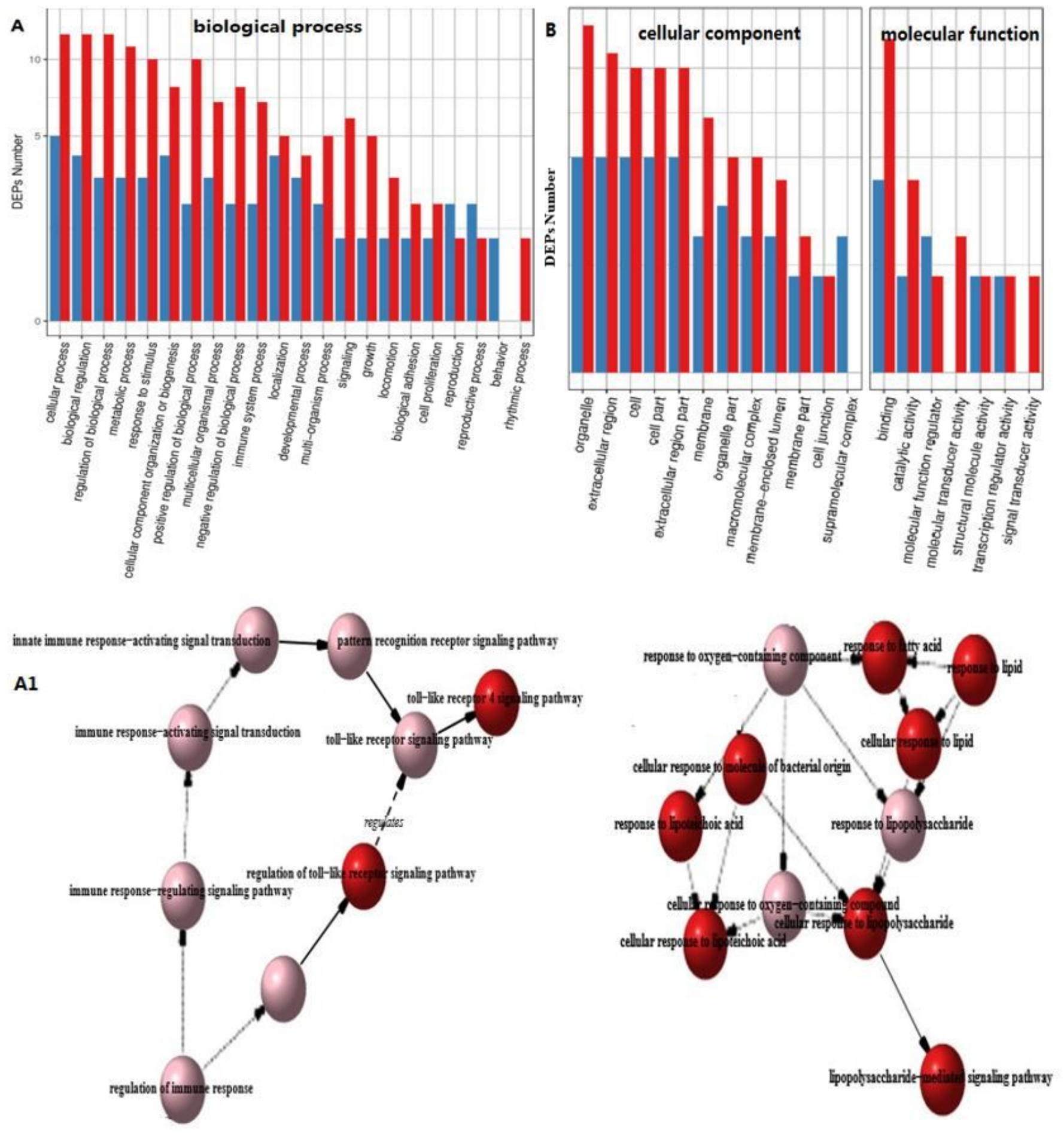

Figure 2

Gene ontology functional classification of plasma-derived exosomes in rheumatoid arthritis. A: Classification of biological process, where red histogram represents upregulated proteins and blue histogram represents downregulated proteins. B: Classification of cellular component and molecular function. A1: The major immune-related networks based on biological process annotation, where red balls represent upregulated pathway. 


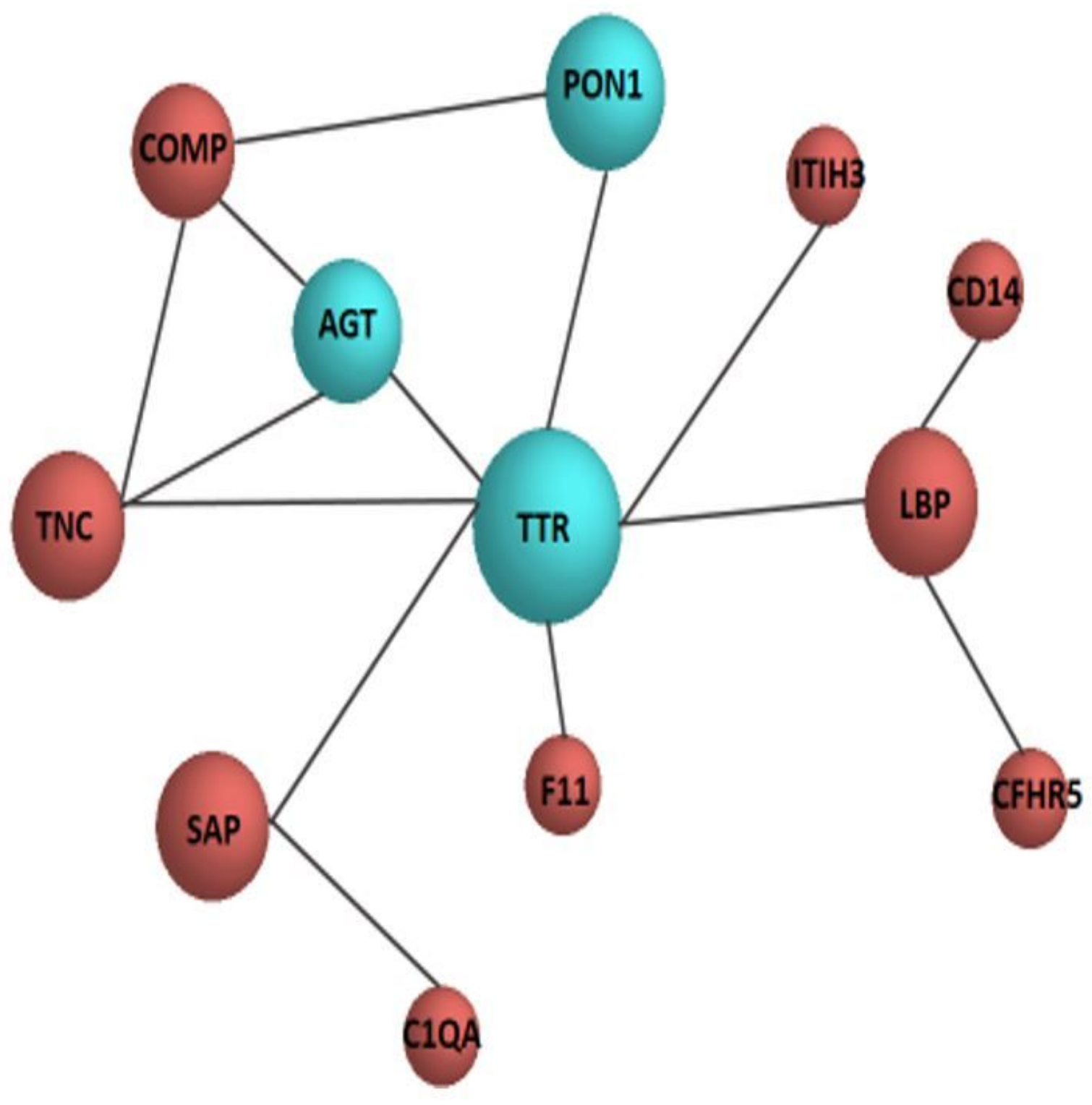

\section{Figure 3}

Network interaction diagram of differentially expressed exosomal proteins of rheumatoid arthritis patients compared with STRING Protein Library. Red circle: upregulated proteins. Blue circle: downregulated proteins. The size of the circle indicates the intensity of relationship. TTR: Transthyretin; AGT: Angiotensinogen; PON1: Serum paraoxonase/arylesterase 1; COMP: Cartilage oligomeric matrix protein; TNC: Tenascin; SAP: Serum amyloid P; LBP: Lipopolysaccharide-binding protein; CD14: Monocyte differentiation antigen CD14; F11: Coagulation factor XI; ITIH3: Inter-alpha-trypsin inhibitor heavy chain H3; CFHR5: Complement factor H-related protein 5; C1QA: Complement C1q subcomponent subunit A. 


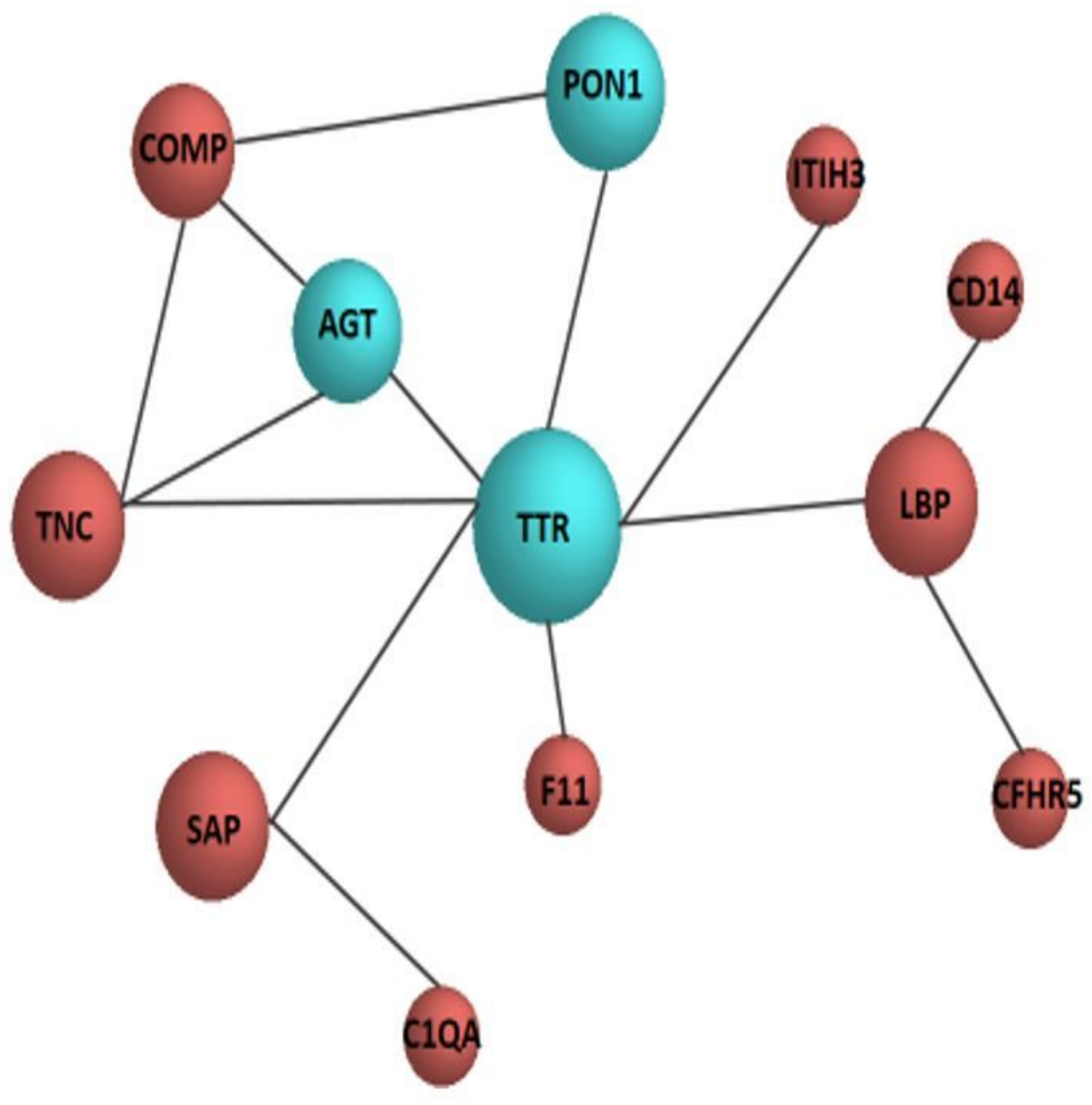

\section{Figure 3}

Network interaction diagram of differentially expressed exosomal proteins of rheumatoid arthritis patients compared with STRING Protein Library. Red circle: upregulated proteins. Blue circle: downregulated proteins. The size of the circle indicates the intensity of relationship. TTR: Transthyretin; AGT: Angiotensinogen; PON1: Serum paraoxonase/arylesterase 1; COMP: Cartilage oligomeric matrix protein; TNC: Tenascin; SAP: Serum amyloid P; LBP: Lipopolysaccharide-binding protein; CD14: Monocyte differentiation antigen CD14; F11: Coagulation factor XI; ITIH3: Inter-alpha-trypsin inhibitor heavy chain H3; CFHR5: Complement factor H-related protein 5; C1QA: Complement C1q subcomponent subunit A. 


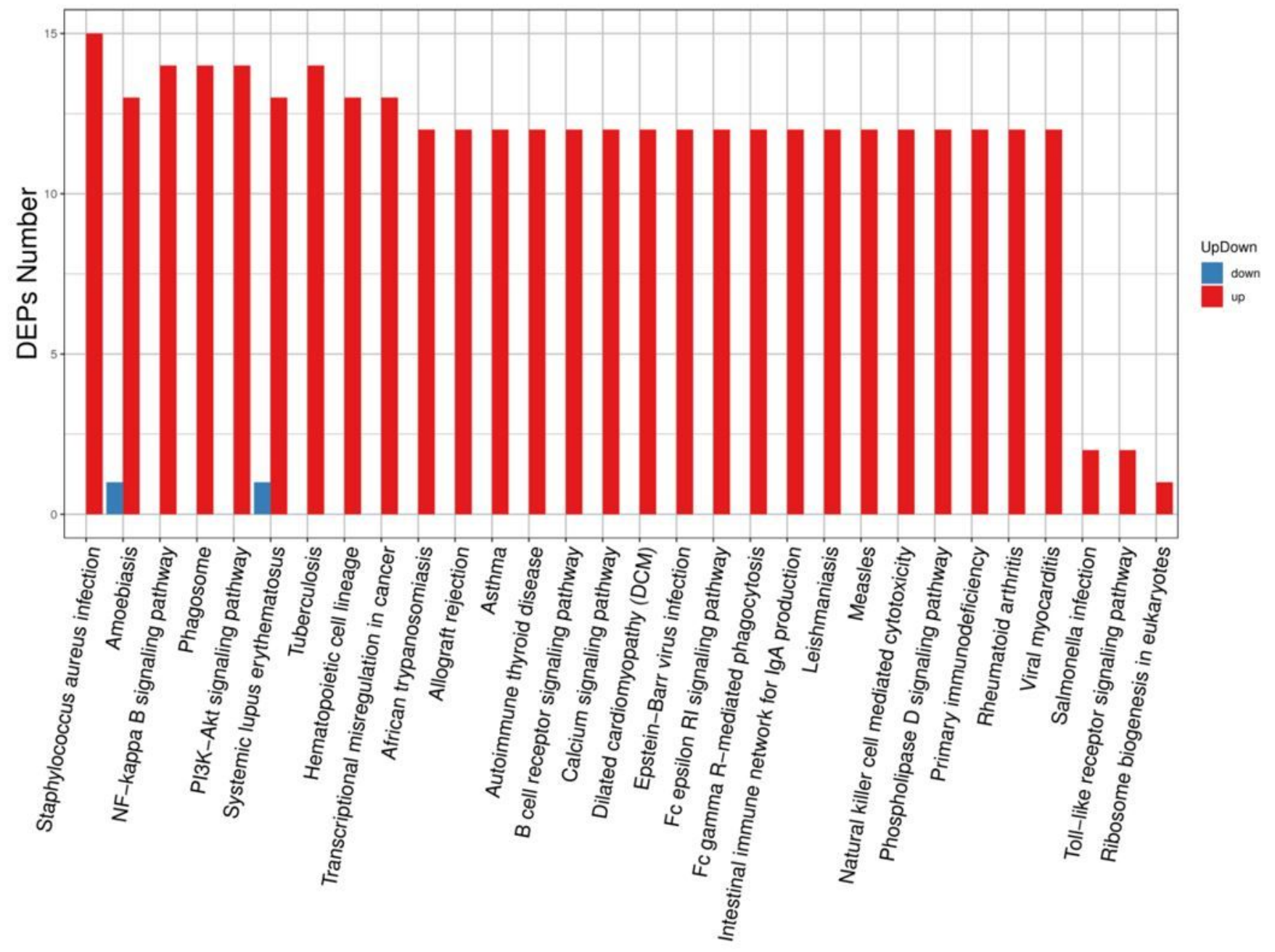

Figure 4

Pathway analysis of differentially expressed proteins (DEPS) in rheumatoid arthritis. 


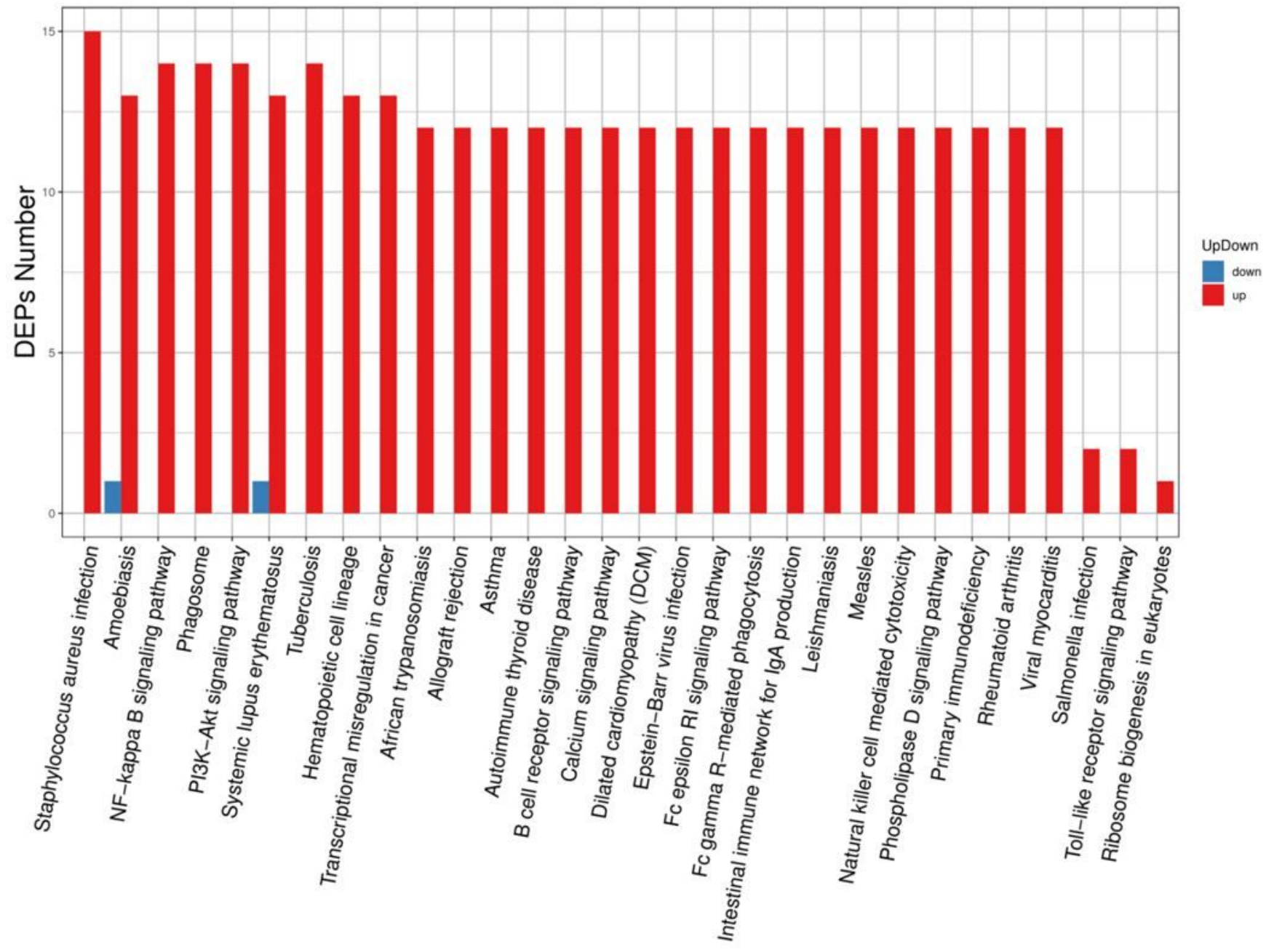

Figure 4

Pathway analysis of differentially expressed proteins (DEPS) in rheumatoid arthritis. 


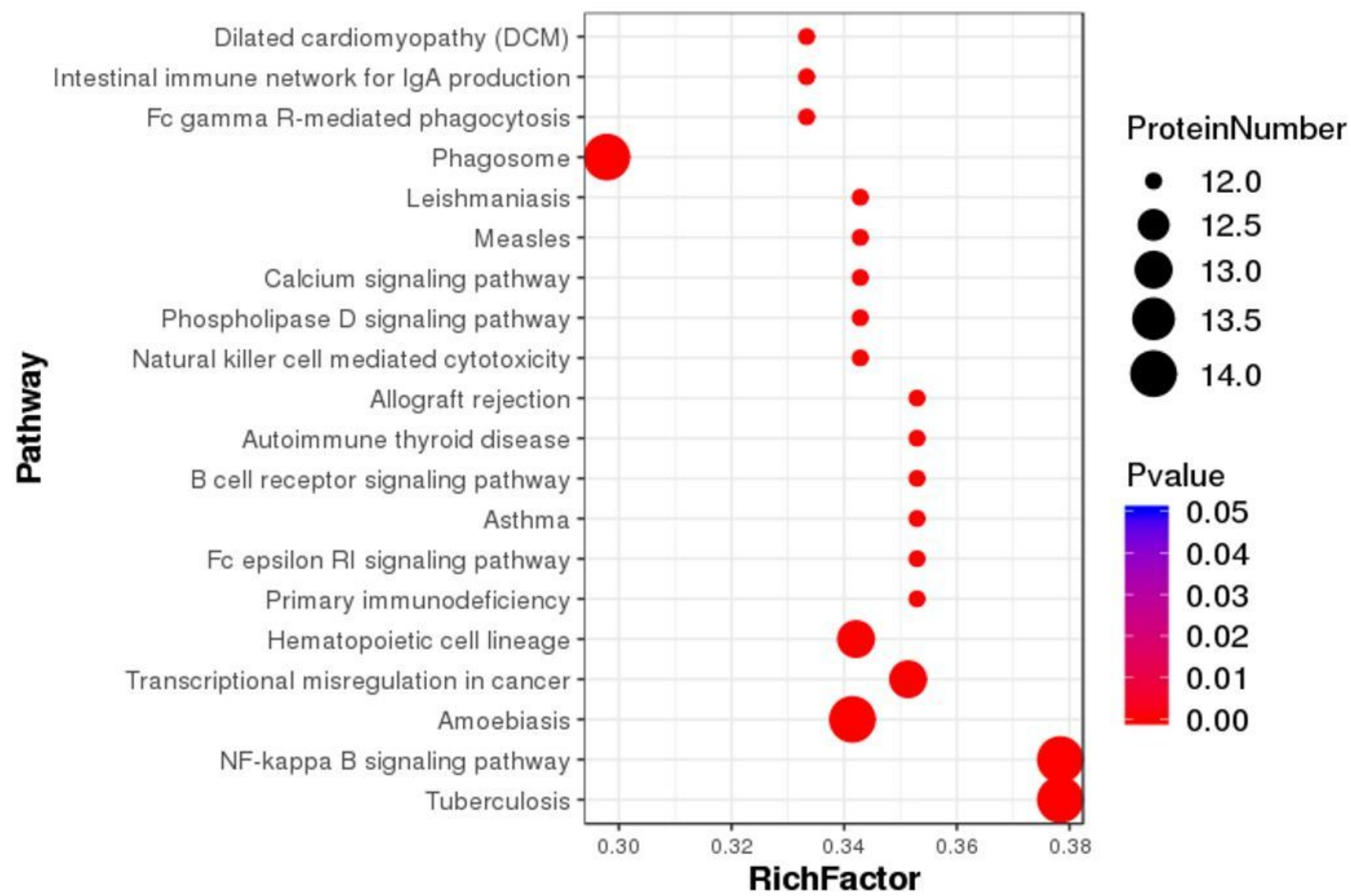

Figure 5

Integrated analysis based on metabolic pathways enrichment of differentially expressed proteins (DEPS) in rheumatoid arthritis patients compared with normal controls. *Rich factor: DEPs annotated to pathway/all identified proteins annotated to pathway. The dot size represents the number of DEPs annotated to pathway. 


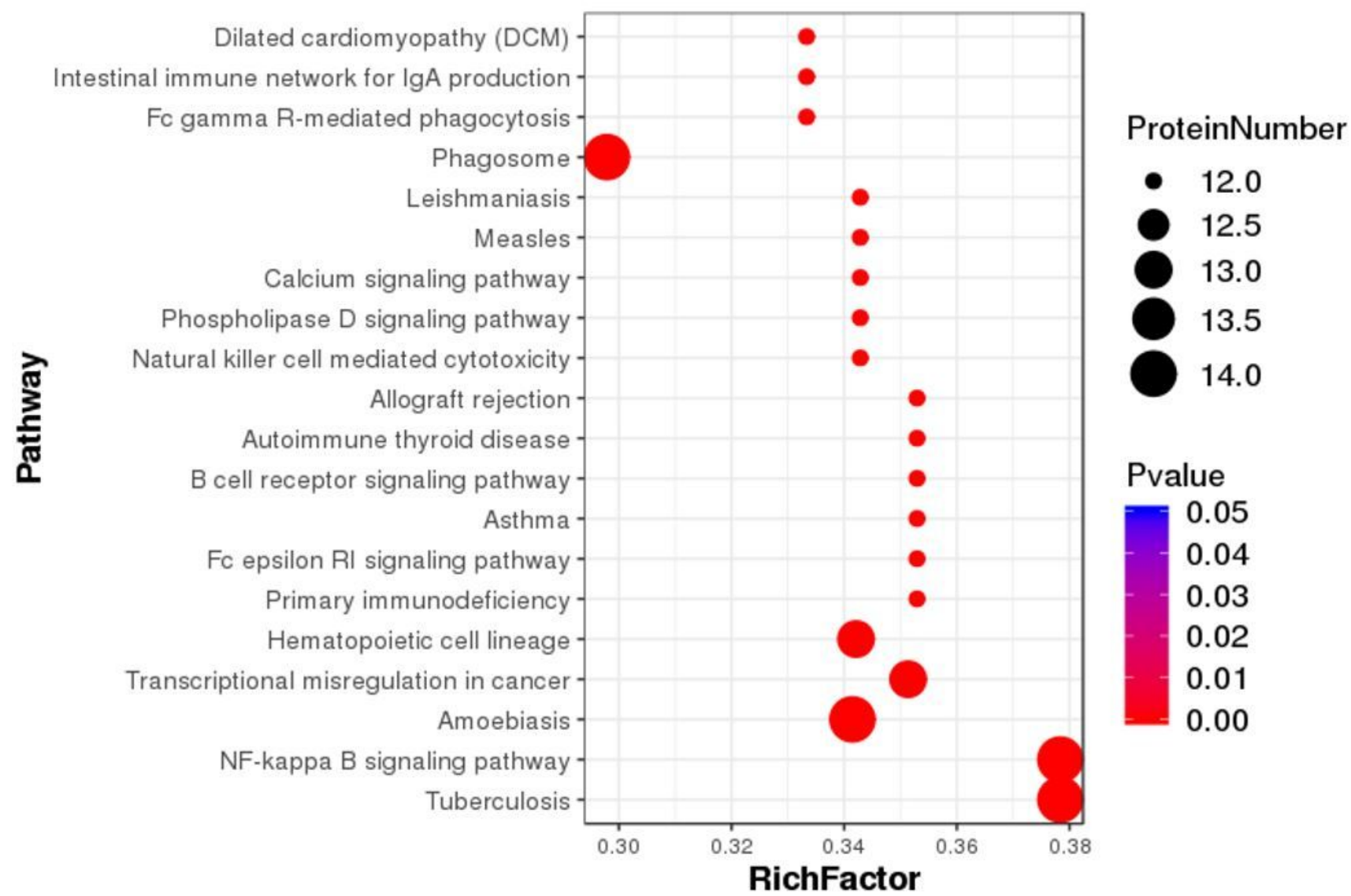

Figure 5

Integrated analysis based on metabolic pathways enrichment of differentially expressed proteins (DEPS) in rheumatoid arthritis patients compared with normal controls. *Rich factor: DEPs annotated to pathway/all identified proteins annotated to pathway. The dot size represents the number of DEPs annotated to pathway. 


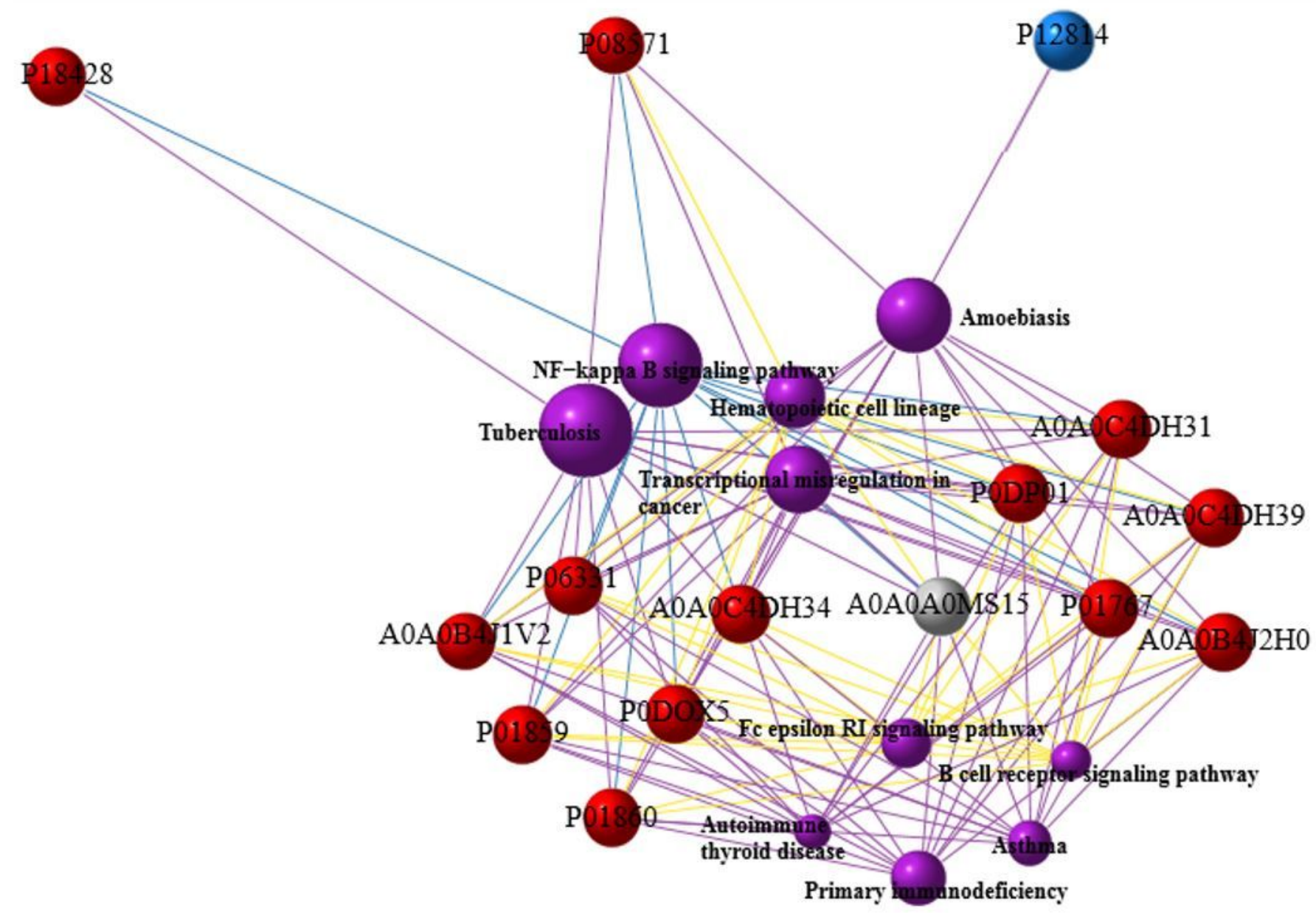

\section{Figure 6}

Integrate analysis based on pathways network enrichment of differentially expressed proteins (DEPS) in rheumatoid arthritis compared with normal controls. *Red ball: upregulated DEP. Blue ball: downregulated DEP. Purple ball: the top 10 enrichment pathways. Larger balls with more markers indicate more enrichment. Different colored lines represent different classification of pathways: red-cellular processes, blue-environmental information processing, green-genetic information processing, purple-human diseases, orange-metabolism, yellow-organismal systems, brown-drug development. P18428: lipopolysaccharide-binding protein (LBP); P08571: monocyte differentiation antigen CD14 (CD14); P12814: alpha-actinin-1 (ACTN1); P06331: immunoglobulin heavy variable 4-34 (IGHV4-34); P01859: immunoglobulin heavy constant gamma 2 (IGHG2); P01860: IGHG3; P0DOX5: immunoglobulin gamma-1 heavy chain (IGY1HC); P01767: IGHV3-53; P0DP01: IGHV1-8; A0A0C4DH31: IGHV1-18; A0A0C4DH39: IGHV1-58; A0A0B4J2H0: IGHV1-69D; A0A0C4DH34: IGHV4-28; A0A0B4J1V2: IGHV2-26; A0A0A0MS15: IGHV3-49. 


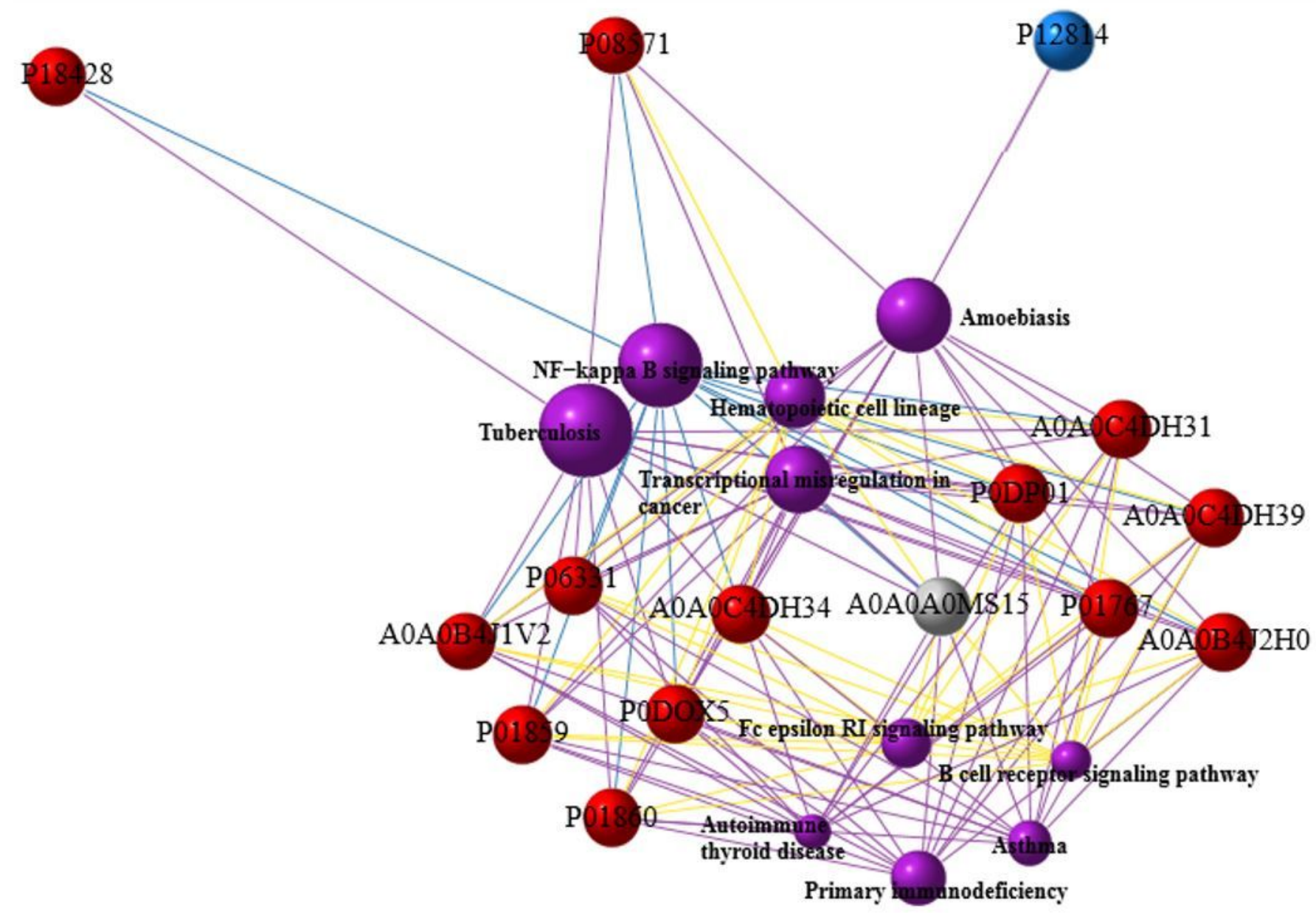

\section{Figure 6}

Integrate analysis based on pathways network enrichment of differentially expressed proteins (DEPS) in rheumatoid arthritis compared with normal controls. *Red ball: upregulated DEP. Blue ball: downregulated DEP. Purple ball: the top 10 enrichment pathways. Larger balls with more markers indicate more enrichment. Different colored lines represent different classification of pathways: red-cellular processes, blue-environmental information processing, green-genetic information processing, purple-human diseases, orange-metabolism, yellow-organismal systems, brown-drug development. P18428: lipopolysaccharide-binding protein (LBP); P08571: monocyte differentiation antigen CD14 (CD14); P12814: alpha-actinin-1 (ACTN1); P06331: immunoglobulin heavy variable 4-34 (IGHV4-34); P01859: immunoglobulin heavy constant gamma 2 (IGHG2); P01860: IGHG3; P0DOX5: immunoglobulin gamma-1 heavy chain (IGY1HC); P01767: IGHV3-53; P0DP01: IGHV1-8; A0A0C4DH31: IGHV1-18; A0A0C4DH39: IGHV1-58; A0A0B4J2H0: IGHV1-69D; A0A0C4DH34: IGHV4-28; A0A0B4J1V2: IGHV2-26; A0A0A0MS15: IGHV3-49. 